\title{
Trabalho e saúde na população transexual: fatores associados à inserção no mercado de trabalho no estado de São Paulo, Brasil
}

\author{
Work and health issues of the transgender population: factors \\ associated with entering the labor market in the state of São Paulo, \\ Brazil
}

Maria Aparecida da Silva (https://orcid.org/0000-0001-5356-3075) ${ }^{1}$

Carla Gianna Luppi (https://orcid.org/0000-0001-9183-8594) ${ }^{2}$

Maria Amélia de Sousa Mascena Veras (https://orcid.org/0000-0002-1159-5762) ${ }^{3}$

${ }^{1}$ Centro de Referência e Treinamento em DST-HIV/ Aids, Programa Estadual de DST-HIV/Aids do Estado de São Paulo, Secretaria de Estado da Saúde. R. Santa Cruz 81, Vila Clementino. 04121-000 São Paulo SP Brasil.

cida@crt.saude.sp.gov.br ${ }^{2}$ Universidade Federal de São Paulo. São Paulo SP Brasil.

${ }^{3}$ Faculdade de Ciências Médicas, Santa Casa de São Paulo. São Paulo SP Brasil.

\begin{abstract}
The transgender population encounters several barriers in entering the labor market, whichfurther impacts their health.In this cross-sectional study, the factors associated with their insertion in the formal job market were analyzed. Participants were recruited in seven municipalities of the state of São Paulo between 2014 and 2015. Logistic regression was used to estimate the odds ratios (OR) of the association between formal insertion and selected characteristics. We included 672 individuals, of which $82.3 \%$ were working, $13.1 \%$ were not employed, and $4.6 \%$ were not in the labor market. Among those working, only $16.7 \%$ were in the formal labor market. Factors independently associated with formal employment were: being atrans man, having 12 years or more of education, being under follow-up for gender transition procedures in the healthcare services, having private health insurance, and never having been arrested. Our results highlightpart of the obstacles the transgender population has toovercome to enter the labor market, and point to the need for public policies specifically designed for the transgender population aiming to reduce school dropout and expand access to comprehensive health care, thereby reducing theirvulnerability.
\end{abstract}

Key words Transgender people, Labor market, Stigma, Discrimination
Resumo A população transexual enfrenta diversas barreiras para sua inserção no mercado de trabalho, com impacto sobre a sua saúde. Neste estudo foram analisados fatores associados à sua inserção no mercado de trabalho formal. Trata-se de estudo transversal conduzido em sete municípios do estado de São Paulo entre 2014 e 2015. Foi estimado "odds ratio" (OR) da associação entre inserção formal e características selecionadas. Foram incluídos 672 indivíduos, 82,3\% com algum vínculo de trabalho, 13,1\% não ocupados e 4,6\% fora do mercado de trabalho. Entre os ocupados, apenas $16,7 \%$ encontravam-se no mercado de trabalho formal. Os fatores associados independentemente ao vínculo formal de trabalho foram: identidade de gênero homem trans, ter 12 anos ou mais de estudo, estar realizando a transição de gênero em serviço de saúde, ter seguro de saúde privado e nunca ter sido preso na vida. Os nossos resultados revelam parte dos obstáculos superados por uma pequena parcela da população de pessoas transexuais para se inserir no mercado de trabatho formal e apontam para a necessidade de politicas públicas desenhadas especificamente para a população transexual, visando a redução do abandono escolar e ampliação do acesso à saúde integral, reduzindo assim sua vulnerabilidade.

Palavras-chave Pessoas transexuais, Mercado de trabalho, Estigma, Discriminação 


\section{Introdução}

A população de pessoas transexuais enfrenta barreiras para o acesso às instituições formais da sociedade como saúde e trabalho ${ }^{1-6}$. No nível estrutural existe carência de políticas públicas voltadas para a sua inclusão no conjunto das instituições sociais, como a educação, o sistema de saúde e o mercado de trabalho. Altos níveis de desemprego e a dificuldade para inserção no mercado de trabalho são também associadas ao estigma e à discriminação vivenciados por esta população ${ }^{7,8}$.

Além de ser reconhecido como um direito humano fundamental ${ }^{9}$, o trabalho constitui-se em uma estratégia para o indivíduo produzir os meios para o seu sustento e possui repercussões diretas sobre as condições de vida e de saúde de qualquer população $0^{10-12}$, sendo considerado um importante determinante social de saúde ${ }^{13}$.

No Brasil, os contextos econômico, social e político, acompanhados de mudanças na estrutura produtiva, não asseguram a todos os trabalhadores a proteção que a legislação faculta ${ }^{14-16}$, fazendo com que coexistam duas modalidades de inserção no trabalho: uma formal e outra informal. O mercado de trabalho formal é prerrogativa dos indivíduos assalariados, que se encontram amparados por um conjunto de leis trabalhistas e medidas de proteção. $\mathrm{O}$ mercado de trabalho informal compreende um conjunto de atividades que as pessoas desenvolvem sem amparo da legislação trabalhista ${ }^{11,14-17}$.

Existem poucos estudos que investigam a inserção no mercado de trabalho de pessoas transexuais no Brasil e no Mundo, em geral são exploratórios e restritos ao contexto da investigação das Infecções Sexualmente Transmissíveis (IST), em especial pelo HIV ${ }^{5,18-21}$.

Dispor de dados sobre a inserção da população de travestis e transexuais no mercado formal de trabalho no estado de São Paulo pode identificar a magnitude do problema e fornecer contribuições na formulação de políticas públicas. O objetivo deste estudo foi analisar os fatores associados à inserção no mercado formal de trabalho em pessoas transexuais no estado de São Paulo.

\section{Métodos}

Foram analisados dados de um estudo transversal, denominado "Vulnerabilidades, demandas de saúde e acesso a serviços da população de travestis e transexuais do Estado de São Paulo" - "Projeto Muriel”22.
O projeto estudou uma amostra de 673 transexuais que acessaram serviços de saúde e assistência social situados em sete municípios do estado de São Paulo (São Paulo, Campinas, São Bernardo do Campo, Santo André, Santos, São José do Rio Preto e Piracicaba). O tamanho da amostra foi calculado por meio da realização de um mapeamento sobre o número de pessoas travestis e transexuais matriculadas ou que frequentaram ou participaram de alguma atividade desenvolvida pelo serviço de saúde e assistência social, no ano de 2012, nos municípios previamente selecionados para o estudo.

A estratégia de amostragem combinou uma abordagem consecutiva de travestis e transexuais, clientes desses serviços, com a técnica conhecida como bola de neve, que tem por base as redes de relações sociais. Os critérios de inclusão adotados foram: ter 16 anos ou mais na ocasião da entrevista; identificar-se como travesti, transexual ou transgênero e residir no estado de São Paulo há menos de seis meses.

O instrumento de coleta de dados foi composto por sete blocos de questões contendo informações: sociodemográficas, percurso profissional, condições de saúde, saúde sexual e reprodutiva, violação de direitos humanos e discriminação, contexto social e percurso de transição. O questionário foi aplicado em entrevista face a face, por meio de um tablet e entrevistador treinado. A coleta de dados teve início no segundo semestre de 2014 e término em janeiro de 2015. Para esta análise foram utilizados dados com respostas válidas para as questões relacionadas à situação de trabalho.

A ocupação das pessoas participantes foi investigada por meio das seguintes perguntas: "Você trabalha atualmente?"; e se trabalha, "Qual é o tipo de vínculo empregatício"; se não trabalha, "Como você vive?" "Pra você tem sido difícil encontrar emprego?" Foram considerados ocupados todos que informaram estar trabalhando atualmente. Os ocupados foram classificados em formal ou informal, de acordo com o tipo de vinculo informado (Figura 1). Foi considerado trabalhador formal aqueles que declararam serem empregados com carteira assinada, funcionários públicos municipais, estaduais ou militares, estagiários remunerados e empregadores com CNPJ. Foram classificados como trabalhadores informais indivíduos ocupados sem carteira de trabalho assinada, que relataram trabalhar por conta própria e empregadores sem CNPJ. Essas categorias foram adaptadas a partir da classificação utilizada pelo IBGE na Pesquisa Nacional por Amostra de Domicilio Continua-PNAD ${ }^{23}$. 


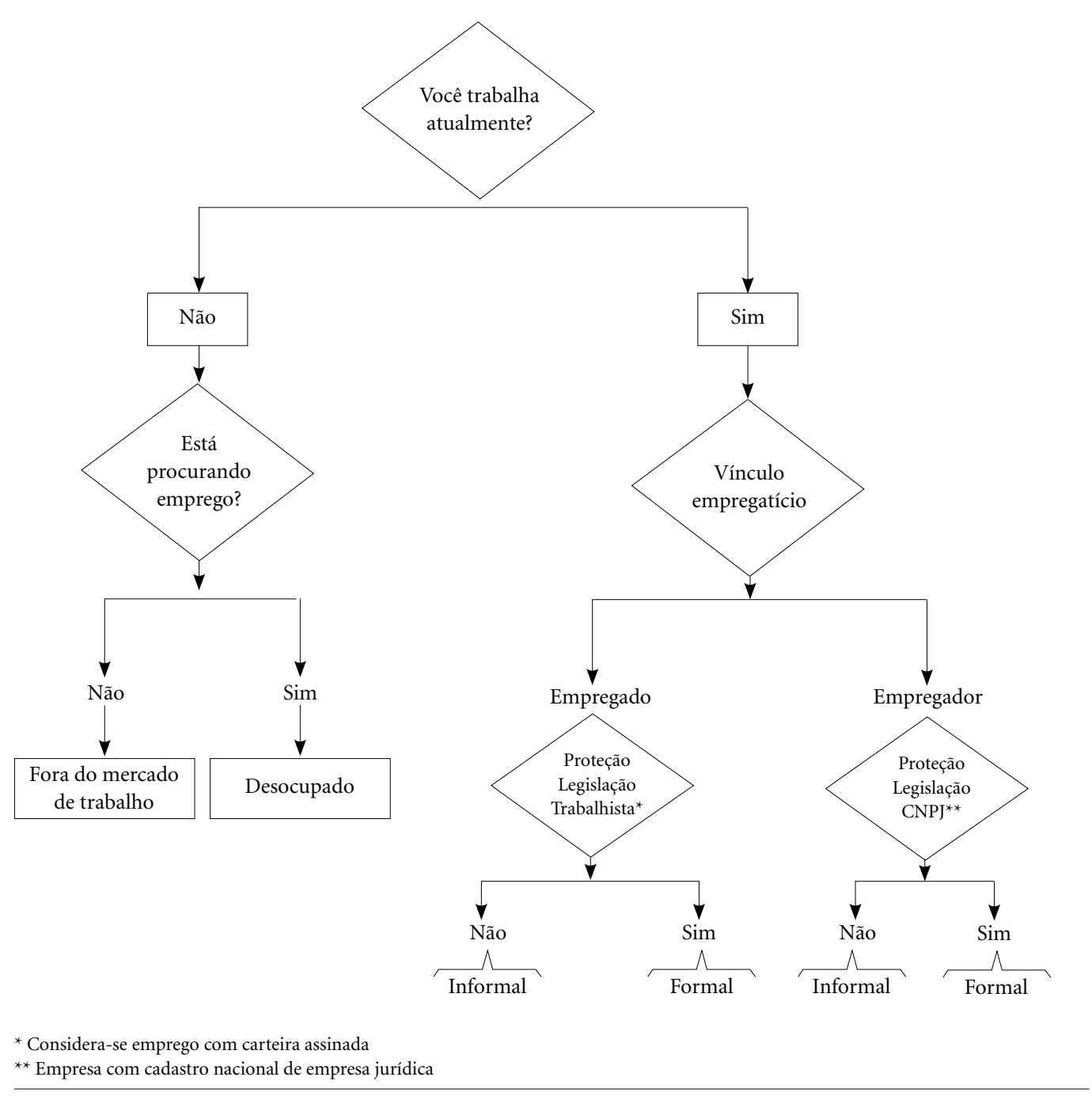

Figura 1. Fluxograma para a definição da situação de ocupação.

Fonte: Própria

A variável dependente foi inserção no mercado formal (sim, não). As variáveis independentes foram: identidade de gênero (mulher transexual/travesti, homem transexual), anos de estudo (menor ou igual a 8 anos, 9 a 11 e 12 anos e mais), faixa etária em anos (16 a 24, 25 a 39, 40 e mais ), raça/cor da pele (branca, preta, parda, amarela, indígena, ignorado), mudou alguma vez da cidade de nascimento ( $\operatorname{sim}$, não), mudança de nome em algum documento ( sim, não, ignorado), possui formação ou curso técnico/profissionalizante ( $\operatorname{sim}$, não), já se sentiu discriminado ( $\operatorname{sim}$, não), já foi preso alguma vez na vida (sim, não), tipo de acompanhamento médico (não faz nenhum acompanhamento, seguimento de aids ou Infec- ções Sexualmente Transmissíveis (IST), seguimento para processo transexualizador e outros), realiza algum acompanhamento médico (sim, não); Realiza acompanhamento para processo transexualizador (sim, não), possui convênio/ seguro de saúde particular ( $\operatorname{sim}$, não).

Foi realizada uma análise descritiva das variáveis. A caracterização do grupo de pessoas ocupadas foi conduzida por meio de análise bivariada e multivariada. Foram calculados os odds ratios (OR) brutos e ajustadas (ORaj) para cada variável independente, com os respectivos intervalos de confiança de 95\% (IC 95\%), utilizando-se o modelo de regressão logística. As variáveis foram testadas para compor o modelo múltiplo quan- 
do apresentaram valor de $\mathrm{p}<=0,20$ na análise bivariada, em ordem crescente (forward stepwise selection). Para a permanência no modelo foi considerado um intervalo de confiança de 95\% que não incluísse a nulidade $(\mathrm{OR}=1,00)$, obtido por meio do teste da razão da máxima verossimilhança. A adequação do modelo foi verificada com o teste de Hosmer e Lemeshow (goodness-of$f i t)$. Todas as análises estatísticas foram realizadas com o auxílio do software STATA ${ }^{\oplus}$, versão 13.0.

$\mathrm{O}$ projeto foi desenhado e conduzido em consonância com as normas de ética em pesquisas com seres humanos no Brasil. Tendo sido submetido e aprovado pelos CEP do CRT/DST AIDS, da SMS-SP e da SMS Santo André. Os participantes assinaram o Termo de Consentimento Livre e Esclarecido (TCLE). Pessoas diagnosticadas com algum problema de saúde foram encaminhadas para serviços de saúde ou assistência social no âmbito dos serviços públicos para tratamento e acompanhamento.

\section{Resultados}

Da amostra total de 673 , foram analisadas as entrevistas de 672 participantes com informações válidas. Dos 672 incluídos, $82,3 \%$ se encontravam ocupados, $13,1 \%$ não ocupados e $4,6 \%$ fora do mercado de trabalho (Tabela 1). Com relação à posição na ocupação, observou-se que 53,9\% exerciam atividades por conta própria e $27,2 \%$ como empregados, mas apenas $14 \%$ deles tinham contratos protegidos pela legislação trabalhista. Atuavam como trabalhadores do sexo $40,6 \%$ dos entrevistados (Tabela 1).

As identidades de gênero relatadas foram: homem transexual $(7 \%)$, mulher transexual ou travesti $(90,4 \%)$, e em $2,5 \%$ da amostra $(n=17)$ essa informação estava ignorada.

Em relação às características demográficas: a média de idade foi 32 anos, 50,4\% estava na faixa etária de 25 a 39 anos, $62 \%$ se autoidentificaram como pertencentes à raça/cor da pele branca, $16,2 \%$ tinham 12 anos ou mais de estudo, $60,5 \%$ moravam em residência própria ou alugada. $\mathrm{O}$ local de residência atual da maioria das pessoas entrevistadas $(49,5 \%)$ era o município de São Paulo. Quanto às características relacionadas à formação profissional, seguridade social, experiência de encarceramento e uso de serviços de saúde, observou-se que: $56,8 \%$ realizaram algum curso técnico/profissionalizante, $37,9 \%$ contribuem para o Instituto Nacional de Seguridade social (INSS), $25,4 \%$ tinham antecedente de pri-
Tabela 1. Inserção no mercado de trabalho de uma amostra de pessoas transexuais de sete municípios do estado de São Paulo, 2014 a 2015.

\begin{tabular}{|c|c|c|}
\hline \multirow{2}{*}{$\begin{array}{r}\text { Variáveis } \\
\text { Condição na Ocupação }\end{array}$} & \multicolumn{2}{|c|}{ Total } \\
\hline & \multirow[b]{2}{*}{553} & \multirow[b]{2}{*}{82,3} \\
\hline Ocupado & & \\
\hline Não ocupados & 88 & 13,1 \\
\hline Fora do mercado de trabalho & 31 & 4,6 \\
\hline \multicolumn{3}{|l|}{ Posição na ocupação } \\
\hline Conta própria & 362 & 53,9 \\
\hline Empregado & 183 & 27,2 \\
\hline Empregador & 4 & 0,6 \\
\hline Estagiário & 2 & 0,3 \\
\hline Não ocupado & 119 & 17,7 \\
\hline Ignorado & 2 & 0,3 \\
\hline \multicolumn{3}{|l|}{ Ocupação segundo CBO } \\
\hline $\begin{array}{l}\text { Trabalhadores dos serviços, } \\
\text { vendedores do comércio em lojas e } \\
\text { mercados* }^{*}\end{array}$ & 410 & 61,0 \\
\hline $\begin{array}{l}\text { Trabalhadores de serviços } \\
\text { administrativos }\end{array}$ & 39 & 5,8 \\
\hline $\begin{array}{l}\text { Profissionais das ciências e das } \\
\text { artes }\end{array}$ & 19 & 2,8 \\
\hline Técnicos de nível médio & 17 & 2,5 \\
\hline $\begin{array}{l}\text { Trabalhadores da produção de } \\
\text { bens e serviços industriais }\end{array}$ & 15 & 2,2 \\
\hline $\begin{array}{l}\text { Trabalhadores da produção de } \\
\text { bens e serviços químicos }\end{array}$ & 6 & 0,9 \\
\hline $\begin{array}{l}\text { Membros superiores do poder } \\
\text { público, dirigentes de organizações } \\
\text { de interesse público }\end{array}$ & 3 & 0,4 \\
\hline $\begin{array}{l}\text { Trabalhadores de manutenção e } \\
\text { reparação }\end{array}$ & 3 & 0,4 \\
\hline Setor agropecuário & 1 & 0,1 \\
\hline Não ocupado & 119 & 17,7 \\
\hline Ignorado & 40 & 6,0 \\
\hline \multicolumn{3}{|l|}{$\begin{array}{l}\text { Tempo de trabalho na atividade em } \\
\text { anos }\end{array}$} \\
\hline Menos de dois anos & 67 & 10,0 \\
\hline De dois a três anos & 94 & 14,0 \\
\hline Mais de três anos & 340 & 50,6 \\
\hline Não ocupado & 119 & 17,7 \\
\hline Ignorado & 52 & 7,7 \\
\hline \multicolumn{3}{|l|}{ Mercado Formal } \\
\hline Não & 459 & 68,3 \\
\hline Sim & 94 & 14,0 \\
\hline Não ocupado & 119 & 17,7 \\
\hline \multicolumn{3}{|l|}{ Trabalhadores do Sexo } \\
\hline Não & 280 & 41,7 \\
\hline Sim & 273 & 40,6 \\
\hline Não ocupado & 119 & 17,7 \\
\hline Total & 672 & 100,0 \\
\hline
\end{tabular}


são alguma vez na vida, 13\% estavam realizando acompanhamento médico para o chamado processo transexualizador e $16,3 \%$ possuíam convênio médico. A distribuição das características da amostra está apresentada na Tabela 2.

Para verificar os fatores associados à inserção no mercado formal na análise bivariada foi considerada apenas a população ocupada, excluindo-se as 119 pessoas não ocupadas e outras 25 para as quais as variáveis de interesse estavam em branco ou tinham resposta ignorada (Tabela 3 ).

Tabela 2. Características sociodemográficas de uma amostra de pessoas transexuais de sete municípios do estado de São Paulo, 2014 a 2015.

\begin{tabular}{|c|c|c|}
\hline \multirow{2}{*}{ Variáveis } & \multicolumn{2}{|c|}{ Total } \\
\hline & $\mathbf{N}$ & $\%$ \\
\hline \multicolumn{3}{|l|}{ Cidade de residência atual } \\
\hline São Paulo & 336 & 50,0 \\
\hline Campinas & 104 & 15,5 \\
\hline Grande SP & 56 & 8,3 \\
\hline Interior & 115 & 17,1 \\
\hline Litoral & 58 & 8,6 \\
\hline Ignorado & 3 & 0,4 \\
\hline \multicolumn{3}{|l|}{ Identidade de gênero } \\
\hline Homen trans & 47 & 7,0 \\
\hline Mulher trans/travesti & 608 & 90,5 \\
\hline Ignorado & 17 & 2,5 \\
\hline \multicolumn{3}{|l|}{ Faixa etária em anos } \\
\hline 16 a 24 & 178 & 26,5 \\
\hline 25 a 39 & 339 & 50,4 \\
\hline 40 e mais & 155 & 23,1 \\
\hline \multicolumn{3}{|l|}{ Raça/Cor da Pele } \\
\hline Branca & 253 & 37,6 \\
\hline Parda & 289 & 43,0 \\
\hline Preta & 101 & 15,0 \\
\hline Amarela & 13 & 1,9 \\
\hline Indígena & 13 & 1,9 \\
\hline Sem resposta & 3 & 0,4 \\
\hline \multicolumn{3}{|l|}{ Anos de estudos } \\
\hline Menor ou igual a 8 & 234 & 34,8 \\
\hline 9 a 11 & 329 & 49,0 \\
\hline 12 anos e mais & 109 & 16,2 \\
\hline \multicolumn{3}{|l|}{ Tipo de moradia } \\
\hline Residência provisória & 254 & 37,8 \\
\hline Casa apartamento próprio / alugado & 407 & 60,6 \\
\hline Ignorado & 11 & 1,6 \\
\hline \multicolumn{3}{|l|}{ Mudança de nome em algum documento } \\
\hline Não & 613 & 91,2 \\
\hline Sim & 58 & 8,6 \\
\hline Ignorado & 1 & 0,1 \\
\hline
\end{tabular}

Das 528 pessoas ocupadas, a proporção de vínculo formal foi: 16,7\%. Quando considerada a identidade de gênero, observou-se que entre os homens transexuais a ocupação formal foi de $59,4 \%$, enquanto que entre as mulheres transexuais/travestis foi apenas de 13,9, o que representa uma chance nove vezes mais alta dos homens transexuais estarem inseridos no mercado formal quando comparados com as mulheres transexuais/travestis (Tabela 3).

Em relação às características sociodemográficas, entre os indivíduos com 12 anos e mais de estudo, a chance de conseguir estar no mercado formal foi de quase 19 vezes quando comparados com aqueles cuja escolaridade era igual ou menor a 8 anos de estudo. Na análise bivariada, observou-se também que estar inserido no pro-

Tabela 2. Características sociodemográficas de uma amostra de pessoas transexuais de sete municípios do estado de São Paulo, 2014 a 2015.

\begin{tabular}{|c|c|c|}
\hline \multirow{2}{*}{ Variáveis } & \multicolumn{2}{|c|}{ Total } \\
\hline & $\mathbf{N}$ & $\%$ \\
\hline \multicolumn{3}{|c|}{ Possui formação ou curso técnico/profissionalizante } \\
\hline $\operatorname{Sim}$ & 382 & 56,8 \\
\hline Não & 290 & 43,2 \\
\hline \multicolumn{3}{|c|}{ Renda mensal em salário mínimos (SM) } \\
\hline Menor de um & 186 & 27,7 \\
\hline 1 a 2 & 187 & 27,8 \\
\hline 2 a 3 & 124 & 18,5 \\
\hline Acima de 3 & 128 & 19,0 \\
\hline Ignorado & 47 & 7,0 \\
\hline \multicolumn{3}{|l|}{ Contribui para o INSS } \\
\hline Não & 412 & 61,3 \\
\hline Sim & 256 & 38,1 \\
\hline Ignorado & 4 & 0,6 \\
\hline \multicolumn{3}{|l|}{ Já foi preso alguma vez na vida } \\
\hline Não & 501 & 74,6 \\
\hline Sim & 171 & 25,4 \\
\hline \multicolumn{3}{|l|}{ Tipo de acompanhamento médico } \\
\hline Não faz nenhum acompanhamento & 274 & 40,8 \\
\hline $\begin{array}{l}\text { Seguimento de aids ou infecções } \\
\text { sexualmente transmissíveis (IST) }\end{array}$ & 217 & 32,3 \\
\hline $\begin{array}{l}\text { Seguimento para processo } \\
\text { transexualizador }\end{array}$ & 88 & 13,1 \\
\hline Outros & 93 & 13,8 \\
\hline \multicolumn{3}{|c|}{ Possui convênio médico / seguro particular de saúde } \\
\hline Não & 560 & 83,3 \\
\hline Sim & 110 & 16,4 \\
\hline Ignorado & 2 & 0,3 \\
\hline Total & 672 & 100,0 \\
\hline
\end{tabular}


Tabela 3. Análise bivariada de fatores associados a inserção no mercado formal de trabalho de uma amostra de pessoas transexuais de sete municípios do estado de São Paulo, 2014 a $2015^{\star}$.

\begin{tabular}{|c|c|c|c|c|c|c|c|}
\hline \multirow{3}{*}{ Variáveis } & \multirow{2}{*}{\multicolumn{2}{|c|}{$\begin{array}{c}\begin{array}{c}\text { Mercado } \\
\text { Formal }\end{array} \\
\text { Sim } \\
\end{array}$}} & \multirow{2}{*}{\multicolumn{2}{|c|}{ Total }} & \multirow{3}{*}{$\begin{array}{l}\text { Odds } \\
\text { Ratio } \\
(\text { OR })\end{array}$} & \multirow{3}{*}{$\begin{array}{c}\text { Intervalo } \\
\text { de confiança } \\
\text { (IC) } 95 \%\end{array}$} & \multirow{3}{*}{$\mathbf{p}$} \\
\hline & & & & & & & \\
\hline & $\mathbf{N}$ & $\%$ & $\mathbf{N}$ & $\%$ & & & \\
\hline \multicolumn{8}{|l|}{ Identidade de gênero } \\
\hline Mulher trans/travesti & 69 & 13,9 & 496 & 100,0 & 1,00 & - & \\
\hline Homem transexual & 19 & 59,4 & 32 & 100,0 & 9,04 & $4,27-19,14$ & $<0,000$ \\
\hline \multicolumn{8}{|l|}{ Anos de estudos } \\
\hline Menor ou igual a 8 & 6 & 3,2 & 187 & 100,0 & 1,00 & - & \\
\hline 9 a 11 & 50 & 19,4 & 258 & 100,0 & 7,25 & $3,03-17,30$ & $<0,000$ \\
\hline 12 anos e mais & 32 & 38,6 & 83 & 100,0 & 18,92 & $7,50-47,76$ & \\
\hline \multicolumn{8}{|l|}{ Faixa etária em anos } \\
\hline 16 a 24 & 16 & 11,9 & 134 & 100,0 & 1,00 & - & \\
\hline 25 a 39 & 53 & 19,0 & 279 & 100,0 & 1,72 & $0,94-3,15$ & 0,074 \\
\hline 40 e mais & 19 & 16,5 & 115 & 100,0 & 1,45 & $0,71-2,99$ & 0,302 \\
\hline \multicolumn{8}{|c|}{ Mudou alguma vez da cidade de nascimento } \\
\hline Sim & 61 & 14,6 & 419 & 100,0 & 1,00 & - & \\
\hline Não & 27 & 24,8 & 109 & 100,0 & 1,93 & $1,15-3,22$ & 0,012 \\
\hline \multicolumn{8}{|c|}{ Mudança de nome em algum documento } \\
\hline Não & 76 & 15,8 & 482 & 100,0 & 1,00 & - & \\
\hline $\operatorname{Sim}$ & 12 & 26,1 & 46 & 100,0 & 1,88 & $0,93-3,80$ & 0,077 \\
\hline \multicolumn{8}{|c|}{ Possui formação ou curso técnico/profissionalizante } \\
\hline Não & 26 & 11,5 & 226 & 100,0 & 1,00 & - & \\
\hline Sim & 62 & 20,5 & 302 & 100,0 & 1,98 & $1,21-3,25$ & 0,007 \\
\hline \multicolumn{8}{|l|}{ Já se sentiu discriminado } \\
\hline $\operatorname{Sim}$ & 71 & 15,6 & 455 & 100,0 & 1,00 & - & \\
\hline Não & 17 & 23,3 & 73 & 100,0 & 1,64 & $0,90-2,98$ & 0,105 \\
\hline \multicolumn{8}{|c|}{ Já foi preso alguma vez na vida } \\
\hline $\operatorname{Sim}$ & 5 & 4,0 & 126 & 100,0 & 1,00 & - & \\
\hline Não & 83 & 20,6 & 402 & 100,0 & 6,29 & $2,49-15,90$ & 0 \\
\hline \multicolumn{8}{|c|}{ Realiza algum acompanhamento médico } \\
\hline Não & 25 & 10,8 & 232 & 100,0 & 1,00 & - & \\
\hline Sim & 63 & 21,3 & 296 & 100,0 & 2,23 & $1,358-3,689$ & 0.002 \\
\hline \multicolumn{8}{|c|}{ Realiza acompanhamento para processo transexualizador } \\
\hline Não & 59 & 12,7 & 465 & 100,0 & 1,00 & - & \\
\hline Sim & 29 & 46,0 & 63 & 100,0 & 5,86 & $3,333-10,333$ & $<0,000$ \\
\hline \multicolumn{8}{|c|}{ Possui convênio / seguro de saúde particular } \\
\hline Não & 52 & 11,8 & 440 & 100,0 & 1,00 & - & \\
\hline Sim & 36 & 40,9 & 88 & 100,0 & 5,16 & $3,089-8,638$ & $<0,00$ \\
\hline Total & 88 & 16,7 & 528 & 100,0 & - & - & \\
\hline
\end{tabular}

* Incluídas 528 pessoas transexuais que estavam ocupadas nessa análise.

Fonte: Projeto Muriel

cesso transexualizador apresentou associação com o mercado formal quando comparados com aqueles que não estavam em acompanhamento nenhum (Tabela 3)

No modelo final apresentaram associação independente com a inserção no mercado formal as seguintes características: identidade de gênero homem transexual $\left(\mathrm{OR}_{\mathrm{aj}}=2,7\right.$; $\mathrm{IC}_{\mathrm{aj}} 95 \% 1,7$ $6,5)$, ter 12 anos ou mais de estudo $\left(\mathrm{OR}_{\mathrm{aj}}=7,5\right.$; $\mathrm{IC}_{\mathrm{aj}}$ 95\% 2,7-20,1), estar realizando algum tipo de acompanhamento médico $\left(\mathrm{OR}_{\mathrm{aj}}=1,8 ; \mathrm{IC}_{\mathrm{aj}}\right.$ 95\% 1,0-4,4); estar em acompanhamento para o 
processo transexualizador $\left(\mathrm{OR}_{\mathrm{aj}}=2,1 ; \mathrm{IC}_{\mathrm{aj}} 95 \%\right.$ $1,0-3,4)$; ter seguro de saúde privado $\left(\mathrm{OR}_{\mathrm{aj}}=2,8\right.$ $\mathrm{IC}_{\mathrm{aj}}$ 95\% 1,5-5,1); e nunca ter sido preso na vida $\left(\mathrm{OR}_{\mathrm{aj}}=3,3 ; \mathrm{IC}_{\mathrm{aj}} 95 \% 1,2-8,9\right)$ (Tabela 4).

\section{Discussão}

O percentual da população transexual inserida no mercado formal de trabalho foi extremamente baixo: apenas $16,7 \%$ estavam com vínculo formal. Os fatores associados a possuir um vínculo formal identificados foram: identidade de gênero homem transexual, possuir 12 anos ou mais de escolaridade, estar em algum acompanhamento médico, estar em acompanhamento para processo transexualizador, ter convênio médico particular e nunca ter sido preso na vida.

Ao comparar o percentual de inserção da população transexual no mercado formal de trabalho com os dados da população geral, Pesquisa Mensal de Emprego (PME) do Instituto Brasileiro de Geografia e Estatísticas (IBGE) na região metropolitana de São Paulo, observou-se que o percentual de indivíduos com vínculo formal de trabalho em 2014 e 2015 foi, respectivamente,
$55,3 \%$ e 54,9\%, números muito superiores ao encontrado na população de transexual ${ }^{24}$.

A população de transexual ocupada, mas não incluída no mercado de trabalho formal apresentou uma frequência muito mais elevada que aquela encontrada nas estatísticas relativas à população geral, como a PME, que encontrou $25,9 \%$ da população no mercado informal no ano de $2015^{25}$. Essa diferença parece não ser um fenômeno exclusivamente brasileiro. Em um estudo com a população de pessoas transexuais nos Estados Unidos em 2011, de 6.450 transexuais entrevistadas a frequência de não empregadas, foi o dobro do encontrado na população em ge$\mathrm{ral}^{8}$. Adicionalmente, é possível que os nossos dados também reflitam a conjuntura socioeconômica do Brasil nos anos mais recentes: aumento do percentual de pessoas na informalidade, o que agravaria a desigualdade no que diz respeito às pessoas transexuais, já vítimas de estigma e discriminação e com maior dificuldade de inserção no mercado formal de trabalho ${ }^{18,19}$.

Embora se reconheça que a informalidade no trabalho possa ser uma escolha, deve-se levar em consideração que a falta de contribuição previdenciária torna esse trabalhador ainda mais

Tabela 4. Modelo múltiplo final para os fatores associados a inserção no mercado formal de trabalho de uma amostra de pessoas transexuais de sete municípios do estado de São Paulo, 2014 a $2015^{\star}$.

\begin{tabular}{|c|c|c|c|c|c|}
\hline \multirow{2}{*}{$\begin{array}{l}\text { Variáveis } \\
\text { Identidade de gênero }\end{array}$} & \multirow[t]{2}{*}{$\begin{array}{l}\text { Odds Ratio } \\
\text { (OR) bruto }\end{array}$} & \multirow[t]{2}{*}{$\begin{array}{c}\text { Odds Ratio } \\
\text { (OR) ajustado }\end{array}$} & \multicolumn{2}{|c|}{$\begin{array}{c}\text { IC 95\% } \\
\text { ajustado }\end{array}$} & \multirow[t]{2}{*}{$\mathbf{p}^{*}$} \\
\hline & & & & & \\
\hline Mulher trans/travesti & 1,00 & 1,00 & & & 0,020 \\
\hline Homem transexual & 9,04 & 2,77 & 1,172 & 6,546 & \\
\hline \multicolumn{6}{|l|}{ Anos Estudos } \\
\hline Menor ou igual a 8 & 1,00 & 1,00 & & & \\
\hline 9 a 11 & 7,25 & 4,91 & $1,997 \quad 1$ & 12,0845 & 0,001 \\
\hline 12 e mais & 18,92 & 7,50 & 2,794 & 20,158 & $<0,000$ \\
\hline \multicolumn{6}{|c|}{ Realizando algum acompanhamento médico } \\
\hline Não & 1,00 & 1,00 & & & \\
\hline Sim & 2,23 & 1,87 & 1,015 & 3,447 & 0,044 \\
\hline \multicolumn{6}{|c|}{ Esta realizando acompanhamento para processo transexualizador } \\
\hline Não & 1,00 & 1,00 & & & \\
\hline Sim & 5,86 & 2,14 & 1,058 & 4,364 & 0,034 \\
\hline \multicolumn{6}{|c|}{ Possui convênio / seguro de saúde partiular } \\
\hline Não & 1,00 & 1,00 & & & $<0,000$ \\
\hline Sim & 5,16 & 2,86 & 1,588 & 5,179 & \\
\hline \multicolumn{6}{|l|}{ Já foi preso alguma vez na vida } \\
\hline Sim & 1,00 & 1,00 & & & 0,015 \\
\hline Não & 6,29 & 3,36 & 1,268 & 8,950 & \\
\hline
\end{tabular}

* $\mathrm{P}$ ajustado

Fonte: Projeto Muriel 
vulnerável ${ }^{11,25}$. A contribuição previdenciária, cuja finalidade é garantir alguma segurança e tranquilidade em caso de afastamento do trabalho, seja por aposentadoria, doença, invalidez ou desemprego, no Brasil é prerrogativa para os trabalhadores com carteira assinada, sendo menos frequente entre os não ocupados e com ocupação não formal ${ }^{11,17}$. No presente estudo, dentre os que atuam na informalidade, próximo de $80 \%$ não contribuíram com a previdência, bem superior à encontrada para a população geral, uma média anual de $22,0 \%$, em 2014 e $2015^{24}$. Valores semelhantes aos nossos foram encontrados por Bonassi em Santa Catarina: $74 \%$ de transexuais ou travestis relataram não contribuir para a previdência ${ }^{19,24}$.

Observou-se que a população transexual não ocupada apresentou dificuldades financeiras: $37,8 \%$ moravam em residências provisórias e mais da metade relatou viver com renda mensal inferior a dois salários mínimos. O rendimento médio na população geral no ano de 2014 e 2015 na região metropolitana de São Paulo foi um pouco mais que três salários mínimos à época ${ }^{24}$.

A ocupação relatada por $40 \%$ dos entrevistados neste estudo foi o trabalho sexual, o que corrobora com achados de outros estudos ${ }^{18,26}$. Embora o trabalho sexual conste na Classificação Brasileira de Ocupações - $\mathrm{CBO}^{27}$, sua atuação não é regulamentada no Brasil, ou seja, essa atividade é exercida totalmente na informalidade. A inserção no mercado como trabalhador (a) sexual é provavelmente um dos resultados do estigma de que são vítimas as pessoas transexuais, o que motiva o abandono precoce da escola e a dificuldade de profissionalização por baixa escolaridade. Além disso, a população de mulheres transexuais trabalhadoras do sexo é extremamente vulnerável, em especial, às infecções sexualmente transmissíveis, constituindo-se uma das populações mais afetada globalmente pela infecção do $\mathrm{HIV}^{28-30}$.

Homens transexuais apresentaram maior probabilidade de estarem inseridos no mercado de trabalho formal do que mulheres transexuais. Pode-se inferir sobre uma eventual influência da geralmente alta passabilidade - aparência em concordância com a identidade de gênero com a qual se identifica - dos homens transexuais após o uso de hormônios, que os protegeria de serem identificados como pessoas transexuais, e, consequentemente, poderiam sofrem menor discriminação.

Estudos apontam também que a desigualdade de gênero que persiste no ambiente de trabalho das pessoas cis gênero é reproduzida na esfera da população transexual ${ }^{24}$. Corroborando esta hipótese, investigação realizada nos EUA mostrou que mesmo após a transição de gênero, a probabilidade de mulheres transexuais encontrarem emprego é menor ${ }^{8}$. Segundo relatos de mulheres transexuais ou travestis entrevistadas em Salvador, as dificuldades para a inserção no mercado de trabalho formal eram muito maiores após a transição de gênero ${ }^{31}$. Em contrapartida, homens transexuais, após a transição de gênero, apresentaram maior possibilidade de entrada no mercado, assim como obtiveram melhora na renda ${ }^{32-34}$. Especificamente o estudo de Davidson ${ }^{33}$, demonstrou que mulheres transexuais são mais discriminadas no momento da contratação, recebendo mais recusa em serem contratadas do que homens transexuais. Essa diferenciação também se estendeu para outras categorias da análise, como piores emprego e ganhos salariais, sempre com resultados negativos para as mulheres transexuais ${ }^{33}$.

O percentual de transexuais entrevistados com nível superior em nosso estudo foi de apenas $16,2 \%$, enquanto na população em geral da região metropolitana de São Paulo este percentual no mesmo ano de realização do estudo foi de $66 \%{ }^{24}$. Em outras regiões do Brasil também foi encontrada baixa escolaridade da população de pessoas transexuais. Bonassi et al. ${ }^{19}$, em estudo realizado em Santa Catarina, observou que $33,9 \%(\mathrm{~N}=100)$ de transexuais e travestis abandonaram os estudos entre os 16 e 19 anos de idade. Na região metropolitana de Recife, de 100 travestis incluídas, entre 2008 e 2009, 17,4\% tinham menos de quatro anos de estudos e $44,9 \%$ não chegaram a concluir o ensino fundamental ${ }^{35}$.

É razoável supor que ter 12 anos ou mais de estudo seja um fator positivamente associado à inserção no mercado de trabalho formal. No entanto, este achado não é consistente na literatura: Bauer e Scheim, estudando discriminação e preconceito contra transexuais no Canadá, encontraram uma frequência elevada de transexuais com escolaridade alta, que não estavam inseridos no mercado de trabalho ou não estavam colocados em atividade de acordo com o respectivo grau de qualificação ${ }^{36}$. No Brasil, Rondas e Machado também identificaram que, ainda que seja uma estratégia a ser perseguida, a elevada escolaridade nem sempre garante a inserção dessa população no mercado de trabalho ${ }^{18}$. A baixa escolaridade pode ser explicada, entre outros fatores, por ser a escola um ambiente hostil às pessoas transexuais. Nos EUA, em 2011, transexuais relataram ter sofrido na escola: assédio (78\%), agressão física 
(35\%) e violência sexual $(12 \%)^{8}$, em função de sua identidade de gênero.

Interessante destacar que se encontrou associação entre as variáveis relacionadas ao acesso a cuidados de saúde e a inserção no mercado de trabalho formal: estar realizando acompanhamento para o processo transexualizador e relatar possuir convênio médico privado. É possível que as razões que permitiram estar inserido no limitado número de vagas nos serviços em que são realizados os procedimentos de transição sejam as mesmas pelas quais foram rompidas outras barreiras: como a educação e o acesso ao mercado formal de trabalho. No Brasil, entre 2008 e 2015, dados do Sistema de Informação Hospitalar do Sistema Único de Saúde (SIH-SUS) indicam que foram realizadas apenas 320 cirurgias de processo de transexualizador ${ }^{37}$, havendo longas de filas de espera. Outros estudos também mencionam relações entre o acesso ao mercado de trabalho e os procedimentos de transição de pessoas transexuais. $\mathrm{O}$ estudo PRIDE, da $\mathrm{OIT}^{26}$, observou que entre as dificuldades apontadas por pessoas transexuais para inserção no mercado de trabalho encontravam-se: ter uma expressão de gênero que não se adequa ao nome apresentado em documento oficial de identificação e apresentar transição incompleta por dificuldades em acessar os procedimentos necessários para efetiva- $\mathrm{la}^{26}$. No Brasil, estudo de 2012 observou que entre os diversos motivos para a não realização do processo transexualizador encontravam-se: desvantagens de empregabilidade no mercado formal em decorrência da discriminação da identidade de gênero transexual e insuficiência de recursos financeiros para custear o processo ${ }^{2}$. Há outros possíveis fatores que podem fazer com que os procedimentos de transição de gênero interfiram na inserção profissional: a realização de uma cirurgia de redesignação sexual pode levar a um longo período de afastamento e cuidados ${ }^{38}$. Para aqueles inseridos na formalidade, neste período há garantia dos benefícios da previdência social, isto é, ainda que estejam afastados do trabalho, sua renda se mantém, o que dificilmente ocorre com os ocupados na informalidade.
Nossos achados corroboram as considerações de Almeida et al. ${ }^{39}$, que reforçam a necessidade de levar em conta todos os fatores envolvidos no complexo objeto da saúde e seus determinantes na população transexual, não sendo possível propor intervenções focadas apenas nas manipulações do corpo.

Este estudo apresenta limitações, dentre as quais a seleção dos participantes ter sido realizada em serviços de saúde especializados - entre eles, um ambulatório de saúde integral de travestis e transexuais - e de assistência social, o que poderia resultar em viés de seleção. Outro ponto que deve ser levantado é que o desenho do estudo não foi realizado para investigação da inserção no mercado de trabalho. No entanto, trata-se de um estudo de base populacional com uma amostra grande, e que incluiu pessoas em diferentes regiões do estado de São Paulo, possibilitando levantar um conjunto de informações até então não exploradas para caracterizar acesso ao mercado de trabalho.

Neste estudo foi possível conhecer como se dá a inserção da população de transexuais no mercado de trabalho e os fatores que o favorecem ou dificultam. Destacou-se o baixo percentual de inserção no mercado formal dessa população elevando a sua vulnerabilidade. Dos fatores associados à inserção no mercado de trabalho, torna-se relevante enfatizar as políticas públicas de inclusão escolar, tolerância e respeito à diversidade de identidade de gênero nas instituições de ensino, para reverter o cenário de abandono escolar e consequentemente baixa escolaridade. Outro importante aspecto foi a necessidade de efetivação do acesso ao processo transexualizador no Sistema Único de Saúde, como ação necessária para a redução das desigualdades vivenciadas por transexuais no mercado de trabalho.

Os nossos resultados indicam a necessidade de políticas públicas que tenham como objetivo reduzir o estigma e a discriminação, melhorar o acesso à educação e à qualificação profissional das pessoas transexuais, tornando mais equânime a disputa por uma vaga no mercado de trabalho formal. 


\section{Colaboradores}

MA Silva e CG Luppi trabalharam na análise, interpretação dos dados, redação do artigo e aprovação da versão a ser publicada. MASM Veras trabalhou na concepção, delineamento, análise, interpretação dos dados, redação do artigo e aprovação da versão a ser publicada.

\section{Agradecimentos}

À Fapesp, que financiou o projeto Muriel.

À Secretaria de Saúde do Estado de São Paulo e ao Centro de Referência e Treinamento em Doenças sexualmente transmissíveis - CRT/DST -Aids do Programa Estadual de São Paulo - PESP.

À equipe de profissionais envolvidos no Projeto Muriel, sem os quais não seria possível a efetivação do estudo.

À Márcia Giovanetti (in memoriam) pelo trabalho desenvolvido na promoção da visibilidade, dignidade e direitos humanos de mulheres transexuais e travestis, contribuindo para a formulação de políticas públicas contra o preconceito e a discriminação, e ampliação do acesso aos serviços de saúde para todos.

A todas as pessoas transexuais que participaram do Projeto Muriel.

\section{Referências}

1. Maranhão filho AM. Inclusão de travestis e transexuais através do nome social e mudanças de prenome: Diálogos iniciais com Karen Schawach e outras fontes. Rev Oralidades [internet]. 2012 [acessado 2019 Abr 20]; 11:90-106. Disponível em: http://diversitas. fflch.usp.br/files/5.\%20MARANH\%C3\%83O\%20 FILHO,\%20E.M.A.\%20Inclus\%C3\%A30\%20 de $\% 20$ travestis $\% 20 \mathrm{e} \% 20 \operatorname{tran} 00$ sexuais $\% 20$ atrav\%C3\%A9s\%20do\%20nome\%20social\%20e\%20 mudan\%C3\%A7a\%20de\%20prenome\%20-\%20 di\%C3\%A1logos\%20iniciais\%20com\%20Karen $\% 20$ Schwach\%20e\%20outras\%20fontes_0.pdf.

2. Sampaio LLP, Coelho MTAD. Transexualidade: aspectos psicológicos e novas demandas ao setor saúde. Rev Comunicação, Saúde, Educ 2012; 16(42):637-649.

3. Socías ME, Marshall BD, Arístegui I, Romero M, Cahn $\mathrm{P}$, Kerr T. Factors associated with healthcare avoidance among transgender women in Argentina. Int J Equity Health 2014; 13(1):81.

4. Organização Internacional do Trabalho (OIT), Programa das Nações Unidas para o Desenvolvimento (PNUD), Programa Conjunto das Nações Unidas sobre HIV/AIDS (UNAIDS). Promoção dos direitos humanos de pessoas LGBT no mundo do trabalho: construindo a igualdade de oportunidades no mundo do trabalho, combatendo a homo-lesbo-transfobia [internet]. 2015 [acessado 2019 Abr 20]. Disponível em: https:// issuu.com/pnudbrasil/docs/manual_promocao_direitos_lgbt_mun.

5. Reisner SL, Poteat T, Keatley J, Cabral M, Mothopeng T, Dunham E, Holland CE, Max R, Baral SD. Global health burden and needs of transgender populations: a review. Lancet 2016; 388(10042):412-436.

6. White Hughto JM, Reisner SL, Pachankis JE. Transgender stigma and health: A critical review of stigma determinants, mechanisms, and interventions. Soc Sci Med 2015; 147:222-231.

7. Conron KJ, Scott G, Stowell GS, Landers SJ. Transgender Health in Massachusetts: Results From a Household Probability Sample of Adults. Am J Public Health 2012; 102(1):118-122.

8. Grant JM, Mottet LA, Tanis J, Harrison J, Herman JL, Keisling M. Injustice at every turn: a report of the national transgender discrimination survey, executive summary. Washington: National Center for Transgender Equality and National Gay and Lesbian Task Force; 2011.

9. Organização das Nações Unidas (ONU). Declaração Universal dos Direitos Humanos. Resolução 217 da Assembléia Geral das Nações Unidas. Paris: ONU; 1948. [acessado 2019 Abr 20]. Disponível em: http://www. ohchr.org/EN/UDHR/Documents/UDHR_Translations/eng.pdf

10. Giatti l, Barreto SM. Situação do indivíduo no mercado de trabalho e iniquidade em saúde no brasil. Rev Saude Publica 2006; 40(1):99-106.

11. Ulyssea G. Informalidade no mercado de trabalho brasileiro: uma resenha da literatura. Rev de Economia Política 2006; 26(4):596-618.

12. Miquilin IOC, León LM, Monteiro MI, Filho HRC. Desigualdades no acesso e uso dos serviços de saúde entre trabalhadores informais e desempregados: análise da PNAD 2008, Brasil. Cad Saude Publica 2013; 29(7):1392-1406. 
13. Whitehead M. The concepts and principles of equity in health. Copenhagen: World Health Organization Regional Office for Europe ( [ internet]. 1990; (document number EUR/ICP/RPD 414) [acessado 2018 Abr 20]. Disponível em: http://www.humanitarianweb.org/wp-content/uploads/2017/11/The-concepts-andprinciples-of-equity-and-health.pdf

14. Teodoro M. Características do mercado de trabalho e origem do informal. In: Jaccoud L, Silva FB, Delgado GC, Castro JA, Cardoso-Júnior JC, Theodoro M, Beghin N. Questão Social e Políticas Sociais no Brasil Contemporâneo. Brasília: IPEA; 2005. p. 91-126. [acessado 2018 Abr 20]. Disponível em: http://www.en.ipea.gov. br/agencia/images/stories/PDFs/livros/Cap_3-10.pdf

15. Oliveira HC, Santos JSP, Cruz EFC. O mundo do trabalho: concepções e historicidade. in III Jornada internacional de políticas públicas; 28 a 30 agosto de 2007. São Luiz: Universidade Federal do Maranhão. [acessado 2018 Abr 20]. Disponível em: http://www.joinpp. ufma.br/jornadas/joinppIII/html/Trabalhos/EixoTematicoB/178d5144a74686f5b7ffHILDERLINE\%20 C\%C3\%82MARA_JOSENEIDE\%20SANTOS_EDUARDO\%20CRUZ.pdf

16. Lameiras MAP, Sandro SC. Mercado de trabalho. Conjuntura e Análise. Brasília: IPEA; 2017. [acessado 2018 Abr 20]. Disponível em: http://www.ipea.gov.br/portal/images/stories/PDFs/conjuntura/170418_cc34_ mercado-de-trabalho.pdf

17. Campos AG. Breve histórico das mudanças na regulação do trabalho no Brasil. [internet]. Brasília: IPEA; 2015. [acessado 2019 Abr 20]. Disponível em: http:// repositorio.ipea.gov.br/bitstream/11058/3513/1/td_ 2024.pdf

18. Rondas LO, Machado LRS. Inserção profissional de travestis no mundo do trabalho: das estratégias pessoais às políticas de inclusão. Rev Pesquisas e Práticas Psicossociais 2015; 10(1):194-207.

19. Bonassi BC, Amaral MS, Toneli MJF, Queiroz MA. Vulnerabilidades mapeadas, Violências localizadas: Experiências de pessoas travestis e transexuais no Brasil. Quad Psicol 2015; 17(3):83-98.

20. Rocha RMG, Pereira DL, Dias TM. O contexto do uso de drogas entre travestis profissionais do sexo. Rev Saúde e Sociedade 2013; 22(2):554-565.

21. Roche K, Keith C. Como o estigma afeta o acesso à saúde para profissionais do sexo transgêneros. Rev British Journal of Nursing 2014; 23:1147-1152.

22. Núcleo de Pesquisa em Direitos Humanos e Saúde da População LGBT (NUDHES). Projeto Muriel. [acessado 2018 Dez 28]. Disponível em: https://www.nudhes. com/projeto-muriel

23. Instituto Brasileiro de Geografia e Estatísticas (IBGE). Notas metodológicas: Pesquisa Nacional por Amostra de Domicílios Contínua: Notas Metodológicas. [internet] 2014. [acessado 2019 Abr 20]. Disponível em: ftp:// ftp.ibge.gov.br/Trabalho_e_Rendimento/Pesquisa_ Nacional_por_Amostra_de_Domicilios_continua/ Notas_metodologicas/notas_metodologicas.pdf
24. Instituto Brasileiro de Geografia e Estatísticas (IBGE). Indicadores IBGE: Principais destaques da evolução do mercado de trabalho nas regiões metropolitanas abrangidas pela pesquisa - Ano 2003 a 2015, Recife, Salvador, Belo Horizonte, Rio de Janeiro, São Paulo e Porto Alegre. [internet] 2015. [acessado 2019 Abr 20]. Disponível em: https://ww2.ibge.gov.br/home/estatistica/indicadores/trabalhoerendimento/pme_nova/retrospectiva2003_2015.pdf

25. Organização internacional do Trabalho (OIT). A OIT no Brasil, trabalho descente para uma vida Digna - Escritório do Brasil [ internet]. 2012 [acessado $2018 \mathrm{Abr}$ 20]. Disponível em: http://www.oitbrasil.org.br/sites/ default/files/topic/gender/pub/oit_no_brasil_folder_809.pdf.

26. Organização internacional do Trabalho (OIT). Discrimination at work on the basis of sexual orientation and gender identity: Results of the ILO's PRIDE Project [internet]. 2015. [acessado 2019 Abr 20]. Disponível em: http://www.ilo.org/wcmsp5/groups/public/---dgreports/---gender/documents/briefingnote/ wcms_368962.pdf

27. Brasil. Classificação Brasileira de Ocupações: $C B O$ [internet]. Brasília: MTE; 2010. [acessado 2018 Abr 22]. Disponível em: http://www.mtecbo.gov.br/cbosite/ pages $/$ download?tipoDownload $=3$

28. Soma T, Underhill K. Sex Work and HIV Status Among Transgender Women: Systematic Review and Meta-Analysis. J Acquir Immune Defic Syndr 2008; 48(1):97-103.

29. Baral S, Beyrer C, Muessig K, Poteat T, Wirtz AL, Decker MR, Sherman SG, Kerrigan D. Burden of HIV among female sex workers in low-income and middle -income countries: a systematic review and meta-analysis. Lancet Infect Dis 2012; 12(7):538-549.

30. Programa Conjunto das Nações Unidas (UNAIDS). The Gap Report 2014. [acessado 2019 Mar 9]. Disponível em: http://www.unaids.org/sites/default/files/ media_asset/UNAIDS_Gap_report_en.pdf

31. Magno L, Dourado I, Silva LAV. Estigma e resistência entre travestis e mulheres transexuais em Salvador, Bahia, Brasil. Cad Saude Publica 2018; 34(5): e00135917.

32. Schilt K, Wiswall M. Before and After: Gender Transitions, Human Capital, and Workplace Experiences. The B.E. Journal of Economic Analysis \& Policy Contributions 2008; 8(1). [acessado 2018 Abr 20]. Disponível em: https://www.ilga-europe.org/sites/default/files/ before_and_after_-_gender_transitions_human_capital_and_workplace.pdf

33. Davidson S. Gender inequality: Nonbinary transgender people in the workplace. Rev Cogent Social Sciences [internet]. 2016 [acessado 2019 Abr 20]; 2:1-12. Disponível em: https://scholarworks.umass.edu/cgi/ viewcontent.cgi?referer

34. Geijtenbeek L, Plug E. Is there a penalty for becoming a woman? Is there a premium for becoming a man? Evidence from a sample of transsexual workers. Rev IZA [internet]. 2015 [acessado 2019 Abr 20]; 9077. Disponível em: http://ftp.iza.org/dp9077.pdf 
35. Sousa PJ, Ferreira LOC, Sá JB. Estudo descritivo da homofobia e vulnerabilidade ao HIV/Aids das travestis da Região Metropolitana do Recife. Cien Saude Colet 2013; 18(8):2239-2251.

36. Bauer GR, Scheim AI. Trans PULSE Project Team. Transgender People in Ontario, Canada: Statistics to Inform Human Rights Policy [internet]. London: ON; 2015. [acessado 2019 Abr 20]. Disponível em: https:// www.rainbowhealthontario.ca/wp-content/uploads/woocommerce_uploads/2015/09/Trans-PULSE-Statistics-Relevant-for-Human-Rights-Policy-June-2015.pdf

37. Popadiuk GS, Oliveira DC, Signorelli MC. A Política Nacional de Saúde Integral de Lésbicas, Gays, Bissexuais e Transgêneros (LGBT) e o acesso ao Processo Transexualizador no Sistema Único de Saúde (SUS):avanços e desafios. Cien Saude Colet 2017; 22(5):15091520. [acessado 2019 Mar 10]. Disponível em: https:// doi.org/10.1590/1413-81232017225.32782016

38. Petry AR. Mulheres transexuais e o Processo Transexualizador: experiências de sujeição, padecimento e prazer na adequação do corpo. Revista Gaúcha de Enfermagem 2015; 36(2):70-75. [acessado 2019 Jun 15]. Disponível em: www.scielo.br/rgenf www.seer.ufrgs. br/revistagauchadeenfermagem

39. Almeida GS, Ribeiro ACP, Gebrath Z. As relações de trabalho como um aspecto da assistência à saúde de pessoas trans. In: Coelho MTAD, Sampaio LLP, organizadoras. Transexualidades: um olhar multidisciplinar. Salvador: EDUFBA; 2014. p. 187-200.

Artigo apresentado em 30/05/2019

Aprovado em 07/08/2019

Versão final apresentada em 19/11/2019 